\title{
Using matrix frame to present road traffic injury pattern
}

\author{
Chien-Hsing Wang ${ }^{1,2}$, Wan-Hua Hsieh ${ }^{3}$, Fu-Wen Liang ${ }^{4}$ and Tsung-Hsueh Lu L,6* $^{5}$
}

\begin{abstract}
Background: Although many epidemiological studies have presented road traffic injuries (RTIs) according to the victim's mode of transport, very few have mentioned the mode of transport of the victim's counterparts. We sought to use matrix frame to present the pattern of RTIs based on the International Classification of Diseases, Tenth Revision (ICD-10) codes.

Methods: Patients admitted to Hualien Tzu Chi Hospital, Taiwan, for RTls from January 1, 2013 to December 31, 2016 were included. The numbers and proportions of various crash types of RTIs were presented using a matrix frame. The row margin of the matrix is the second character of ICD-10 codes V00-V79 (victim's mode of transport), and the column margin of the matrix is the third character of ICD-10 codes V00-V79 (mode of transport of victim's counterpart), constituting a 80-cell grid.
\end{abstract}

Results: In total, 2727 patients were included. The cell with the highest proportion in the matrix grid was ICD-10 code V23 "motorcycle rider injured in collision with car, pick-up truck or van" (27.0\%, 737/2727), followed by that of V27 "motorcycle rider injured in collision with fixed or stationary object" (12.5\%, 342/2727) and V28 "motorcycle rider injured in noncollision transport accident" (12.2\%, 334/2727). The matrix pattern of RTls differed with sex and age.

Conclusions: By using the matrix frame, we can easily understand the RTI pattern for different demographic groups and identify the priority crash types.

Keywords: Transport accidents, Road traffic injuries, Pattern of injury, Epidemiology

\section{Background}

Although many epidemiological studies have presented road traffic injuries (RTIs) according to the victim's mode of transport (e.g., pedestrian, bicycle, two-wheel motorcycle, and car) (Chokotho et al. 2013; Majdan et al. 2015; Spoerri et al. 2011; Watson et al. 2015), very few have mentioned the mode of transport of the victim's counterparts, which could provide a more complete picture of the crash event, thus facilitating the design of relevant intervention programs. For instance, in the Netherlands, bicyclists injured in crashes not involving motor vehicles had a higher number of serious injuries than bicyclists injured in crashes involving motor vehicles; in addition, they had

\footnotetext{
* Correspondence: robertlu@mail.ncku.edu.tw

${ }^{5}$ NCKU Research Center for Health Data and Department of Public Health,

National Cheng Kung University, Tainan, Taiwan

${ }^{6}$ Department of Public Health, College of Medicine, National Cheng Kung

University, No. 1, Dah Hsueh Road, Tainan 701, Taiwan

Full list of author information is available at the end of the article
}

different implications for prevention measures, such as the design of bicycle tracks, mobility advice for older bicyclists, and campaigns to encourage bicycle light use (Weijermars et al. 2016).

Compared with the International Classification of Diseases (ICD), Ninth Revision (ICD-9) codes, an innovative feature of the ICD, Tenth Revision (ICD-10) codes for RTI is the use of the mode of transport modular coding frame (Langley and Chalmers 1999), which can be arrayed as a matrix (National Center for Health Statistics 2013). The row margin of the matrix is the second character of ICD-10 codes V00-V79 (victim's mode of transport), and the column margin of the matrix is the third character of ICD-10 codes V00-V79 (the mode of transport of victim's counterpart), constituting a 80-cell grid (Table 1). However, no study thus far has presented the RTI pattern by using a matrix frame. In this study, we sought to use matrix frame 


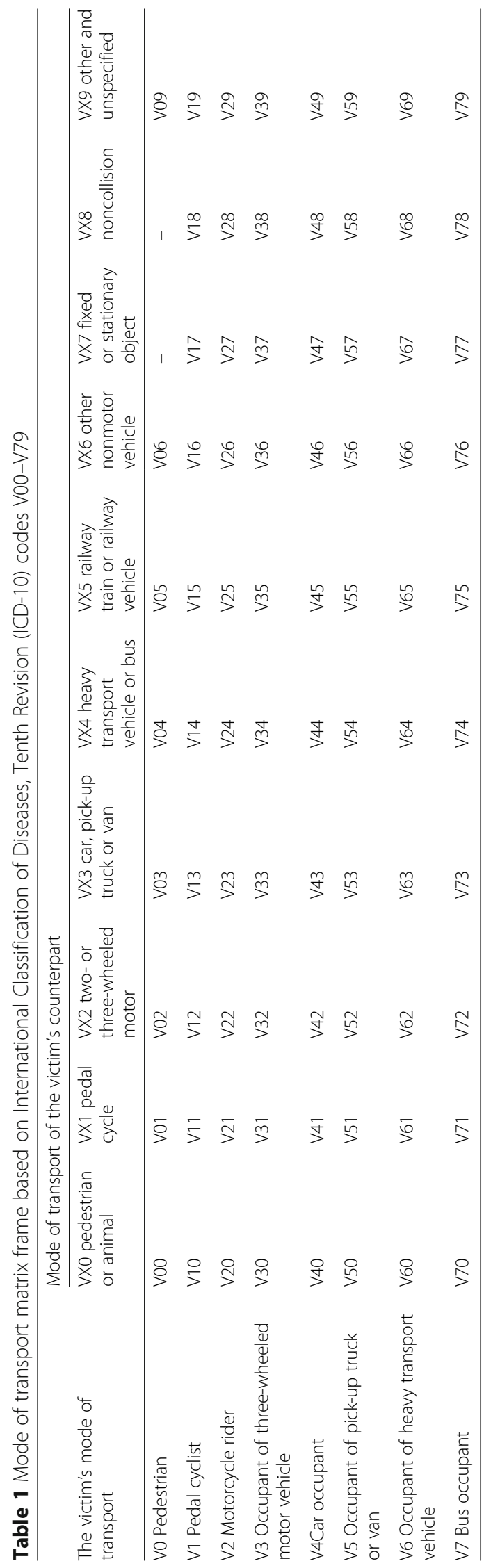


to present the RTI pattern among people admitted to one medical center in Eastern Taiwan affected by RTI.

\section{Methods}

We included patients admitted to Hualien Tzu Chi Hospital, Taiwan, for RTIs from January 1, 2013 to December 31, 2016 and extracted their demographic data (age and sex), ICD-10 codes of external causes of RTIs (V00-V79). The numbers and proportions of various crash types of RTIs were presented using a matrix frame. The users of the matrix can identify the victim's mode of transport (e.g., ICD-10 code V2 motorcycle rider) in row margin first and then the mode of transport of counterpart (e.g., ICD-10 code VX3 car) in column margin and get the number and proportion of cases. To properly interpret of the comparisons of proportions between different crash types, we calculated 95\% confidence intervals for each proportion.

We used the user-friendly self-service business intelligence software Tableau to create a dashboard; therefore, we could select the dimension (specific age group or sex) of our choice. To more clearly visualize the crash types occurring in high proportions, we used a highlighted table: the darker the cell color, the higher the percentage of a particular crash type was. The number and $95 \%$ confidence interval of each proportion are displayed in tooltips that pop out when the user hovers over the mark.

\section{Results}

A total of 2727 patients were included in the analysis. According to the row margin of the matrix (victim's mode of transport: second character of ICD-10 codes V00-V79), the ICD-10 code V2 "motorcycle rider" accounted for the highest proportion (70\%, 1901/2727; Fig. 1); furthermore, the proportions of V2 for victims aged $0-14,15-24,25-44,45-64$, and $>=65$ years were $35 \%, 85 \%, 71 \%, 66 \%$, and $65 \%$, respectively.

In the column margin of the matrix (the mode of transport of victim's counterpart: third character of ICD10 codes V00-V79), ICD-10 code VX3 "car, pick-up truck or van" accounted for the highest proportion (36\%, 906/2516); moreover, the proportions of VX3 for victims aged $0-14,15-24,25-44,45-64$, and $>=65$ years were $38 \%, 41 \%, 32 \%, 34 \%$, and $38 \%$, respectively.

Of the 80 cells in the matrix grid, the cell with highest proportion (darkest color) in the matrix was that of V23 "motorcycle rider injured in collision with car, pick-up truck or van" (27.0\%, 737/2727; Fig. 1), followed by that of V27 "motorcycle rider injured in collision with fixed or stationary object" $(12.5 \%, 342 / 2727)$ and V28 "motorcycle rider injured in noncollision transport accident"

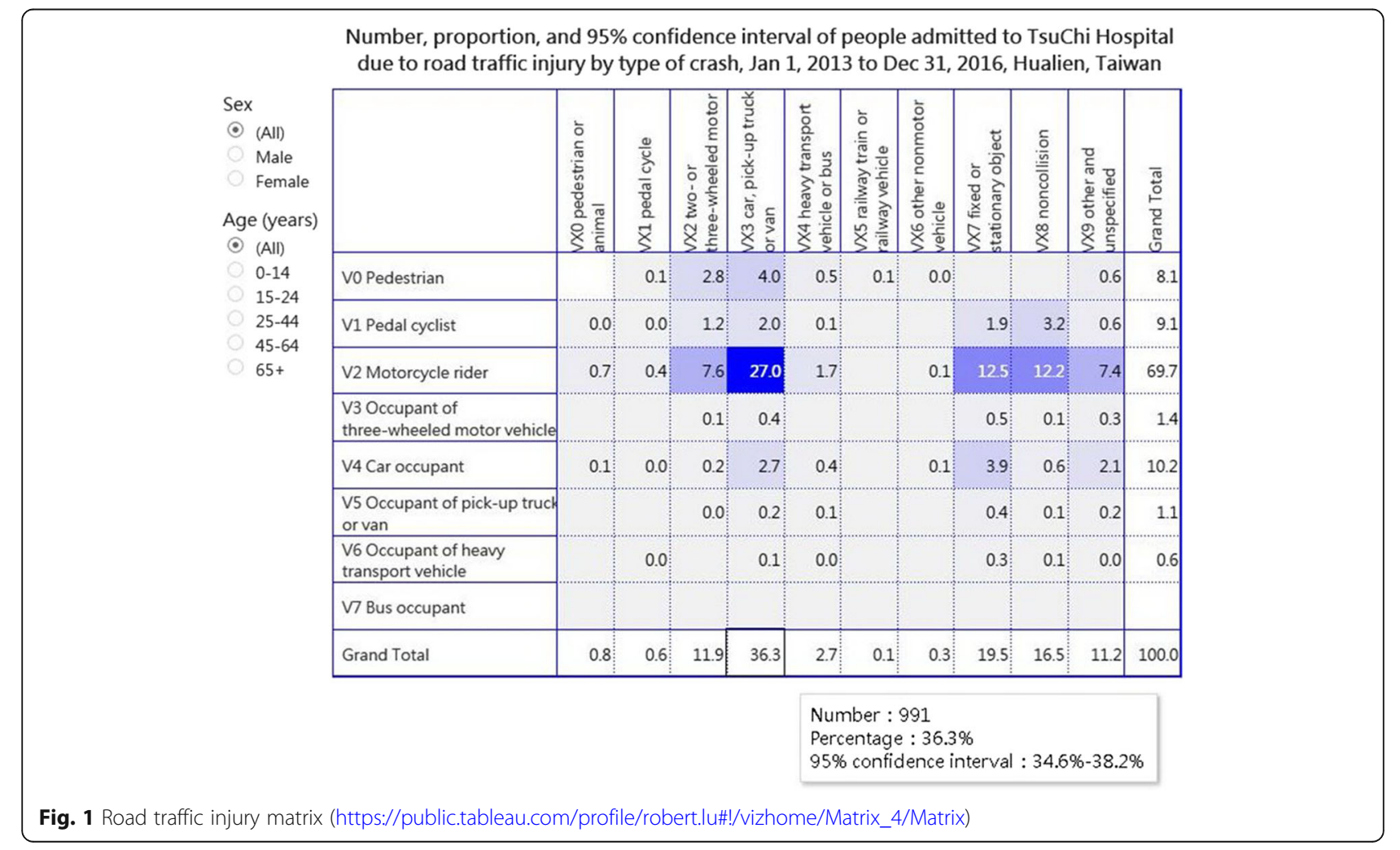


(12.2\%, 334/2727). The proportion of V23 for female patients was $29.5 \%(359 / 1216)$, which is higher than that for male patients $(25.0 \%, 378 / 1511)$.

For patients aged 15-24 years, the cell with the highest percentage of RTIs was that of V23 (36.3\%, $213 / 586)$, followed by that of V27 $(16.6 \%, 97 / 586)$ and V28 (12.6\%, 74/586) (Fig. 2). However, for patients aged $0-14$ years, the cell with the highest percentage of RTIs was that of V03 "pedestrian injured in collision with car, pick-up truck or van" $(14.6 \%, 14 / 96)$, followed by that of V23 $(11.5 \%, 11 / 96)$ and V28 $(10.4 \%$, 10/96) (Fig. 3).

\section{Discussion}

The findings of this study demonstrate that the main mode of transport of RTI victims in Taiwan was the motorcycle, accounting for seven-tenths of all RTIs. By contrast, in the Netherlands, bicycles accounted for three-fifths of all RTIs in 2011 (Weijermars et al. 2016). Therefore, the RTI patterns may differ considerably among countries.

Regarding the mode of transport of the victim's counterpart, the number of injured cyclists in crashes not involving motor vehicles was 5 times the number of injured cyclists in crashes involving motor vehicles in the Netherlands (Weijermars et al. 2016). However, according to our findings, in Taiwan, the number of injured bicyclists in crashes involving motor vehicles (ICD-10 codes V23, V24, and V25) was only 1.5 times the number of injured bicyclists in crashes not involving motor vehicles (ICD-10 codes V20, V21, V26, V27, and V28).

Despite the differences in the RTI pattern between Taiwan and the Netherlands, the proportion of RTIs in vulnerable road users was similar between countries: $88 \%$ in Taiwan and $86 \%$ in the Netherlands. The term "vulnerable road user" refers to people at the highest risk in traffic; these people are not protected by an outside shield, such as pedestrians, bicyclists, and motorcyclists, and have few or no external protective devices to absorb energy in a collision, making them the weak counterpart in a road traffic crash (Costant and Lagarde 2010). Several measures for preventing RTIs among vulnerable road users (e.g., helmet use, conspicuity aids, and avoiding alcohol use) could be applied to motorcyclists in Taiwan and bicyclists in the Netherlands.

Different matrix frame formats have been proposed for presenting injury-related statistics. The most wellknown is the Barell matrix, which details affected body region and nature of injury (e.g., fracture) (Barell et al. 2002; Fingerhut and Warner 2006). Another matrix is the external cause of injury mortality matrix, which details RTIs by the mechanism and intent of injury (McLoughlin et al. 1997; Fingerhut and McLoughlin 2001; Fingerhut 2004; Minino et al. 2006). By using the external cause of injury mortality matrix, we determined that the decrease in the mortality trends of some unintentional injuries might be due to the increase in mortality trends of the same

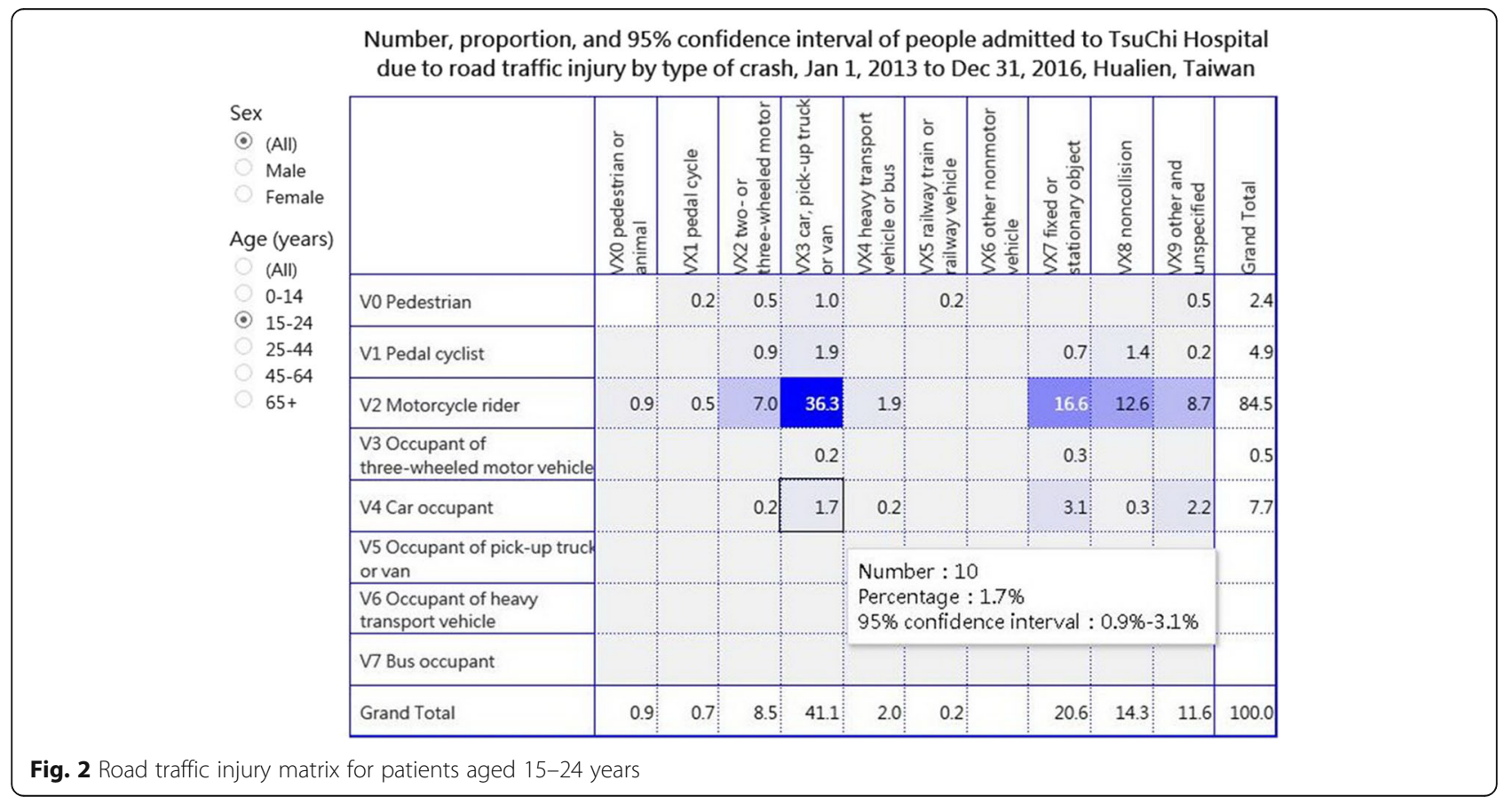




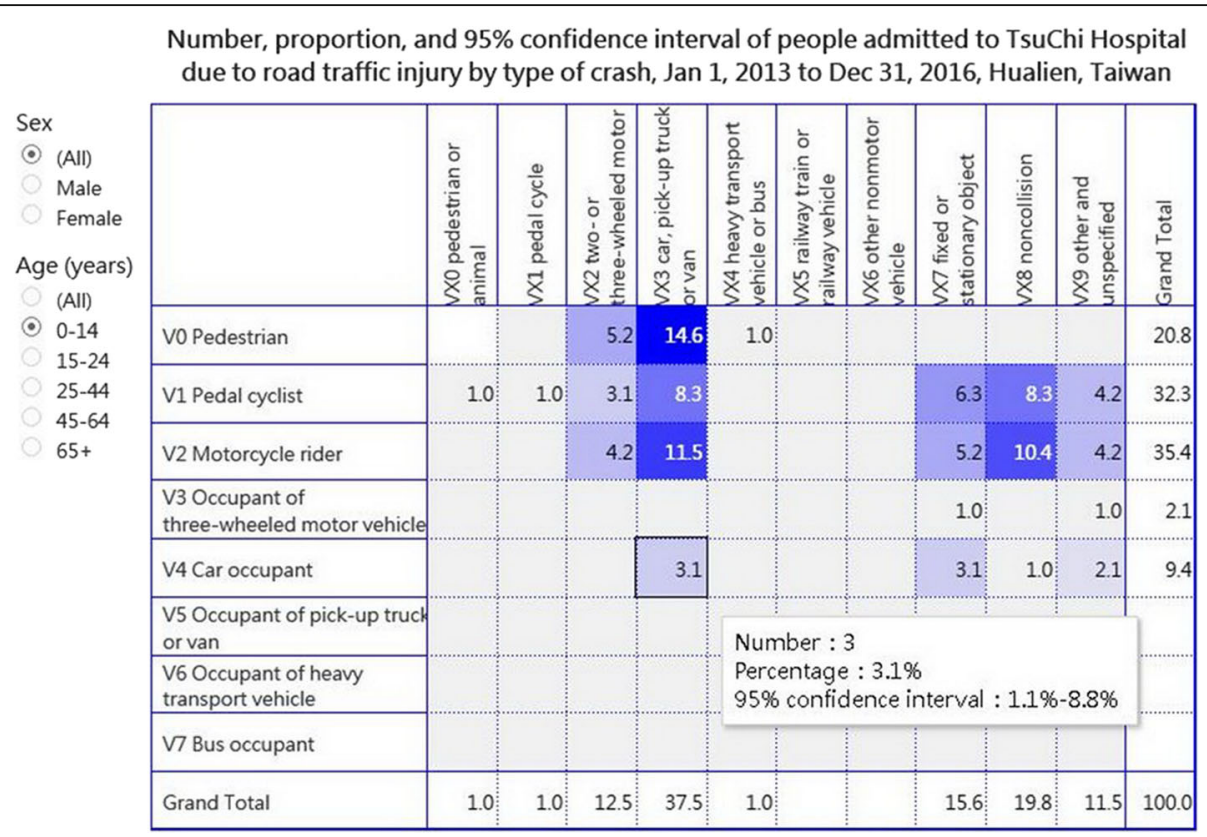

Fig. 3 Road traffic injury matrix for patients aged 14 years and younger

mechanism of injury with an undetermined intent (Lu 2002). However, no study thus far has used the mode of transport matrix frame to present the RTI pattern.

This study has two strengths: (1) the use of the mode of transport matrix frame to present the pattern of RTIs and (2) the use of a visualization dashboard to select the demographic group of choice.

However, this study also has several limitations. First, the mode of transport matrix frame is constructed on the basis of the ICD-10 codes; therefore, if the medical record documentation of the mode of transport is not as specific as required, many RTIs will be classified as "unspecified"; thus, no useful information can be obtained. Nevertheless, the quality of health record documentation on the mode of transport at Hualien Tzu Chi Hospital is relatively high: only $11 \%$ of cases have been classified as ICD-10 code VX9 "other and unspecified". Second, the information of only two dimensions (second and third characters of ICD-10 codes V00-V79) could be presented in the matrix. The information of the fourth character of ICD-10 codes V00-V79 regarding whether the motor vehicle occupant was the driver or passenger and whether it was a traffic or nontraffic accident could not be presented in the same matrix. A solution to this limitation is the use of the drill-down function in the visualization dashboard. Third, because RTIs may be concentrated under particular crash types, the number of RTIs in many cells of the matrix remains zero. In other words, the presentation of the RTI pattern by using a matrix frame may occupy a larger space than that occupied by the traditional presentation method.

\section{Conclusion}

In conclusion, by presenting the mode of transport of both the victim and the victim's counterpart in a matrix frame, we could easily understand the RTI pattern and identify the priority crash types. Studies using matrix frames to compare RTI patterns between countries with different modes of transport are warranted.

\section{Acknowledgements}

The authors thank Ms. Pai-Huan Lin for data visualization.

\section{Authors' contributions}

CW collected and analyzed the data, reviewed the literature and draft the manuscript. WH helped analyzed the data and critical review the manuscript. FL provided crucial statistical suggestions, completed data analysis, and revising the manuscript critically. TL conceived the study, supervise the literature review and analysis and critical review the manuscript. All authors read and approved the final manuscript.

\section{Ethics approval and consent to participate}

This study was approved by the Institutional Review Boards of Chi-Mei Medical Center (10406-003) and TzuChi Hospital (104-67-B).

\section{Competing interests}

The authors declare that they have no competing interests.

\section{Publisher's Note}

Springer Nature remains neutral with regard to jurisdictional claims in published maps and institutional affiliations. 


\section{Author details}

'Division of Plastic Surgery, Department of Surgery and Trauma Center, Hualien Tzu Chi Hospital, Buddhist Tzu Chi Medical Foundation, Hualien, Taiwan. ${ }^{2}$ School of Medicine, Tzu Chi University, Hualien, Taiwan.

${ }^{3}$ Department of Public Health, Tzu Chi University, Hualien, Taiwan. ${ }^{4}$ Department of Public Health, College of Health Sciences, Kaohsiung Medical University, Kaohsiung, Taiwan. ${ }^{5}$ NCKU Research Center for Health Data and Department of Public Health, National Cheng Kung University, Tainan, Taiwan. ${ }^{6}$ Department of Public Health, College of Medicine, National Cheng Kung University, No. 1, Dah Hsueh Road, Tainan 701, Taiwan.

Received: 14 December 2017 Accepted: 5 April 2018

Published online: 23 April 2018

\section{References}

Barell V, Aharonson-Daniel L, Fingerhut LA, et al. An introduction to the Barell body region by nature of injury diagnosis matrix. Inj Prev. 2002;8:91-6.

Chokotho LC, Matzopoulos R, Myers JE. Assessing quality of existing data sources on road traffic injuries (RT/s) and their utility in informing injury prevention in the western Cape Province, South Africa. Traffic Inj Prev. 2013;14:267-73.

Costant A, Lagarde E. Protecting vulnerable road users from injury. PLoS Med. 2010;7:e1000228.

Fingerhut LA. The external cause of injury mortality matrix. Paper presented in WHO family of international classifications network meeting, Reykjavik, Iceland, October 24-30, 2004.

Fingerhut LA, McLoughlin E. Classifying and counting injury. In: Rivara FP, Cummings P, Koipsell TD, et al., editors. Injury control: a guide to research and program evaluation. Cambridge: Cambridge University Press; 2001. p. 15-31.

Fingerhut LA, Warner M. The ICD-10 injury mortality diagnosis matrix. Inj Prev. 2006;12:24-9.

Langley JD, Chalmers DJ. Coding the circumstances of injury: ICD-10 a step forward or backwards? Inj Prev. 1999;5:247-53.

Lu TH. Changes in injury mortality by intent and mechanism in Taiwan, 1975-1998. Inj Prev. 2002;8:70-3.

Majdan M, Rusnak M, Rehorcikova V, Brazinova A, Leitgeb J, Mauritz W. Epidemiology and patterns of transport-related fatalities. Traffic Inj Prev. 2015;16:450-5.

McLoughlin E, Annest JL, Fingerhut L, et al. Recommended framework for presenting injury mortality data. MMWR Recomm Rep 1997;46(No RR-14):1-30.

Minino AM, Anderson RN, Fingerhut LA, Boudreault MA, Warner M. Deaths: injuries, 2002. National vital statistics reports; vol 54 no 10. Hyattsville: National Center for Health Statistics; 2006.

National Center for Health Statistics. Part 2e. Instruction manual-ICD-10 volume 3; 2013. p. 1627-8. Accessed 6 May 2017.at https://www.cdc.gov/nchs/data/ dvs/2e_volume3 2013.pdf.

Spoerri A, Egger M, von Elm E. Mortality from road traffic accidents in Switzerland: longitudinal and spatial analyses. Acc Ana Prev. 2011:43:40-8.

Watson A, Watson B, Vallmuur K. Estimating under-reporting of road crash injuries to police using multiple linked data collections. Acc Ana Prev. 2015;83:18-25.

Weijermars W, Bos N, Stipdonk HL. Serious road injuries in the Netherlands dissected. Traffic Inj Prev. 2016;17:73-9.

\section{Submit your manuscript to a SpringerOpen ${ }^{\circ}$ journal and benefit from:}

- Convenient online submission

- Rigorous peer review

- Open access: articles freely available online

- High visibility within the field

- Retaining the copyright to your article 